\title{
Influence du niveau croissant d'apport d'aliment concentré sur la durée de mastication de la ration chez la vache laitière
}

\author{
JP Dulphy, J Rouel, M Jailler \\ Station de recherches sur la nutrition des herbivores, Centre de recherche Inra de Clermont- \\ Ferrand-Theix, 63122 Saint-Genès-Champanelle, France
}

(Reçu le 12 Avril 1995; accepté le 24 November 1995)

Résumé - Quatre régimes différents, constitués d'un fourrage de base (ensilage d'herbe) et d'apports de concentrés égaux à $0,3,6$ et $9 \mathrm{~kg}$, ont été distribués, dans un schéma expérimental en carré latin, à huit vaches laitières en production. En moyenne les vaches ont ingéré $14,5 \mathrm{~kg}$ de MS d'ensilage et $3,9 \mathrm{~kg}$ de $\mathrm{MS}$ d'aliment concentré, pour un poids vif de $636 \mathrm{~kg}$ et une production de lait à $4 \%$ de MG de $15,8 \mathrm{~kg}$. Les durées journalières de mastication r'ont pas été significativement différentes entre les traitements, soit en moyenne 940 minutes/jour. Par calcul on peut estimer à 10 minutes $/ \mathrm{kg}$ de MS la durée unitaire de mastication de l'aliment concentré et à 62,5 min celle de l'ensilage. Cette dernière valeur ne varie pas significativement avec la proportion de concentré dans la ration. Cet essai confirme que, pour des proportions d'aliment concentré inférieures à $40 \%$, la durée unitaire de mastication du fourrage ne varie pas sensiblement avec le niveau d'apport de l'aliment concentré.

aliment concentré / fourrage / mastication / fibrosité / vache laitière

Summary - Influence of increasing level of concentrate on the chewing time of the ration in dairy cow. Four different diets, made up of a grass silage and four concentrate supplies $(0,3,6,9 \mathrm{~kg})$ were fed, in a latin square design, to eight dairy cows. Average dry matter (DM) intake of the animals was $14.5 \mathrm{~kg}$ for silage and $3.9 \mathrm{~kg}$ for concentrate. Animals had a live weight of $636 \mathrm{~kg}$ and their production was $15.8 \mathrm{~kg}$ fat-corrected milk. The total time of chewing was not significantly different between the treatments (average $940 \mathrm{~min} /$ day). After calculation, it possible to estimate the unitary chewing time of concentrate to $10 \mathrm{~min} / \mathrm{kg} \mathrm{DM}$ and that of forage to $62.5 \mathrm{~min} / \mathrm{kg} D M$; this last value is not influenced by the proportion of concentrate in the diet. This trial confirms that, for proportions of concentrate under $40 \%$, the unitary chewing time of forage can be considered as constant. 


\section{INTRODUCTION}

L'indice de mastication, dont la définition a été proposée par Balch (1971), est une caractéristique originale d'un fourrage donné. Elle traduit pour partie sa «fibrosité», c'està-dire sa résistance à la mastication, et est mesurée par la durée de mastication (ingestion + rumination) ramenée à $1 \mathrm{~kg}$ de $\mathrm{MS}$ ingérée, ou durée unitaire de mastication.

Cet indice de mastication a surtout été utilisé pour les vaches laitières. II ne devrait pas, pour la ration, descendre au-dessous de 30 minutes/kg MS (Sudweeks et al, 1981 ; Nørgaard, 1990), sous peine d'entraîner une chute du taux butyreux ou des problèmes sanitaires (Journet, 1988). L'indice de mastication d'une ration s'obtient, en principe, par addition pondérée des indices du fourrage et du concentré. L'estimation de ces indices pose cependant quelques problèmes. Ainsi on ne peut mesurer directement l'indice de mastication d'un aliment concentré. De plus cet indice dépend un peu du niveau d'ingestion de la ration (Sauvant et al, 1990). Enfin on ne sait pas comment l'indice du fourrage varie avec l'augmentation de l'apport de concentré.

D'après Sudweeks et al (1975) puis Jarrige et al (1995) l'indice de mastication du fourrage ne changerait pas avec un apport croissant de concentré, mais Dulphy et al (1993) observent une très légère influence de cet apport de concentré, chez des vaches laitières. II en est de même pour les moutons (Guérin et Dulphy, 1984 ; Taniguchi et al, 1986). Un essai a donc été mis en place chez ce type d'animal, avec un régime témoin sans concentré, pour examiner si l'apport de concentré modifie, et de combien, l'indice de mastication d'un fourrage de bonne qualité.

\section{MATÉRIEL ET MÉTHODES}

Huit vaches laitières, en deuxième partie de leur lactation et produisant en moyenne $22 \mathrm{~kg}$ de lait à $4 \%$ de MG en début d'essai, ont été utilisées sur le domaine Inra d'Orcival. Les animaux étaient en stabulation entravée, sur litière de paille, mais sans pouvoir en consommer. Le fourrage distribué aux animaux était un ensilage de prairie naturelle préfané, récolté en brins courts et préparé avec addition de 4,1 L/tonne d'acide formique. Ses caractéristiques nutritionnelles sont données dans le tableau I avec celles de l'aliment concentré associé qui était constitué d'un mélange de céréales ( $40 \%$ ), de pulpe de betterave $(30 \%)$ et de tourteaux $(22 \%)$.

Quatre régimes ont été comparés: le fourrage seul (Fo), le même fourrage distribué avec un aliment concentré à raison de 3,6 ou $9 \mathrm{~kg}$ brut (régimes F3, F6, F9). Le fourrage était distribué en une seule fois, à $9 \mathrm{~h}$, après la traite du matin. Le concentré était distribué, non mélangé au fourrage, mais mis sur ce dernier, la moitié à $9 \mathrm{~h} 15$ et l'autre moitiè à $16 \mathrm{~h}$, juste avant la traite du soir.

Tableau I. Composition chimique et valeur nutritive des aliments utilisés.

Ensilage Concentré d'herbe

Teneur en MS $(\%)$

97

En $\mathrm{g} / \mathrm{kg}$ de MS

Matières azotées totales 147

Cendres 123

Ceilulose brute $\quad 300$

NDF

593

$\mathrm{pH}$

$\mathrm{N}-\mathrm{NH}_{3}(\% \mathrm{~N}$ Total $)$

4,19

$\mathrm{N}$-soluble (\% N Total)

7,1

48

En g/kg MS

Acide lactique

Acide acétique

Acide butyrique

Alcools

43

17

1,5

12,3

Valeur nutritive (/ kg MS)

UFL

$0,85 \quad 1,05$

PDIN

$88 \quad 115$

PDIE

80 
La comparaison des quatre régimes a été effectuée dans un schéma expérimental en carré latin (quatre traitements, quatre périodes, deux groupes de quatre animaux). Chaque période a duré 24 ou 25 jours. À la fin de chacune de ces périodes deux séquences de 4 jours ont été consacrées aux mesures des quantités volontairement ingérées et des activités alimentaires des animaux, selon la méthode de Léveillé et al (1979).

Les quantités de MS ingérées ont été mesurées pour les fourrages à partir de celles des quantités offertes et refusées ( $10 \%$ de refus), et corrigées pour les pertes de MS à l'étuve selon la méthode de Dulphy et al (1975). Les quantités de concentré distribuées ont été totalement ingérées. La production de lait a été pesée tous les jours et sa composition analysée 2 jours par semaine. Les animaux ont été pesés à la fin de chaque période.

Les résultats ont été analysés par analyse de variance en distinguant les effets de la période (3 dl), de l'individu (7 dl) et du traitement ( $3 \mathrm{dl}$ ). Seul l'effet du traitement a été considéré pour les résultats (SAS, 1985).

\section{RÉSULTATS}

En moyenne les vaches ont ingéré $14,5 \mathrm{~kg}$ de MS d'ensilage (QIF) et 3,9 kg MS de concentré (QIC) pour un poids vif de $636 \mathrm{~kg}$ et une production de lait à $4 \%$ de MG égale à $15,8 \mathrm{~kg}$. Les quantités de MS de fourrage ingérées ont diminué avec l'apport de concentré (tableau II), les taux de substitution étant respectivement égaux à 0,07 entre $F 0$ et $F 3,0,33$ entre $F 3$ et F6, 0,36 entre F6 et F9.

Malgré une légère diminution avec l'augmentation de l'aliment concentré, les durées journalières de mastication $\left(\mathrm{DM}_{r}\right)$ n'ont pas été significativement différentes entre les traitements $(-5,7 \%$ entre F0 et F9), avec une moyenne de 940 minutes $(65 \%$ du temps journalier), soit 455 pour l'ingestion et 485 pour la rumination.

Si on calcule simultanément les durées unitaires de mastication du fourrage et de l'aliment concentré par régression multiple, on obtient l'équation suivante (QIF et QIC étant respectivement les quantités en $\mathrm{kg}$ de MS de fourrage et de concentré ingérées) :

$$
\begin{aligned}
& \mathrm{DM}_{r}=254+45,9 \mathrm{QIF}+5,7 \mathrm{QIC} \\
& ( \pm 266) \quad( \pm 17,0) \quad( \pm 8,1) \\
& \text { (NS) (S) (NS) } \\
& n=32 \quad \mathrm{ETR}= \pm 109 \quad R^{2}=0,47
\end{aligned}
$$

En forçant le modèle de régression à passer par 0 , on peut estimer, dans le cadre des données utilisées, les influences moyennes respectives du fourrage et du concentré sur la durée totale de mastication :

$$
\begin{aligned}
& \mathrm{DM}_{r}=61,9 \mathrm{QIF}+10,7 \mathrm{QIC} \\
& ( \pm 2,1) \quad( \pm 6,1) \\
& \text { (S) } \quad(p=0,09) \\
& \mathrm{ETR}= \pm 109 \quad n=32
\end{aligned}
$$

Cette équation permet d'estimer à 10 minutes $/ \mathrm{kg}$ MS la durée unitaire de mastication de l'aliment concentré, confirmant l'hypothèse prise par Dulphy et al (1993). Dans ces conditions la durée unitaire de mastication du fourrage ressort en moyenne à 62,5 minutes/kg de MS (tableau II).

Enfin $\mathrm{DM}_{r}$ peut être relié à la quantité de MS ingérée totale (QIT) et à la proportion de concentré $C(0 \leq C<1)$.

$$
\begin{aligned}
& \mathrm{DM}_{r}=\begin{array}{c}
309 \\
( \pm 234)
\end{array}+\begin{array}{rr}
43 \text { QIT } & -813 \mathrm{C} \\
( \pm 15) & ( \pm 259)
\end{array} \\
& \text { (NS) } \quad(p=0,07) \quad \text { (S) [3] } \\
& R^{2}=0,257 \quad \text { ETR }= \pm 107 \quad n=32
\end{aligned}
$$

Il est possible également de relier l'indice de mastication de la ration $\left(\mathrm{IM}_{r}\right)$ à la proportion de concentré :

$$
\begin{array}{ccc}
\mathrm{IM}_{r}=\begin{array}{c}
62,7 \\
( \pm 6,0)
\end{array} & \begin{array}{c}
54,1 \mathrm{C} \\
( \pm 7,6)
\end{array} \quad[4] \\
R^{2}=0,63 & \text { ETR }= \pm 6,0 \quad n=32
\end{array}
$$

Avec cette équation la durée unitaire de mastication du fourrage est estimée à 62,7 minutes $/ \mathrm{kg}$ MS et celle du concentré à 8,6 minutes, soit des valeurs peu différentes de celles estimées par l'équation [2]. 
Tableau II. Quantités de MS ingérées et activités alimentaires des animaux.

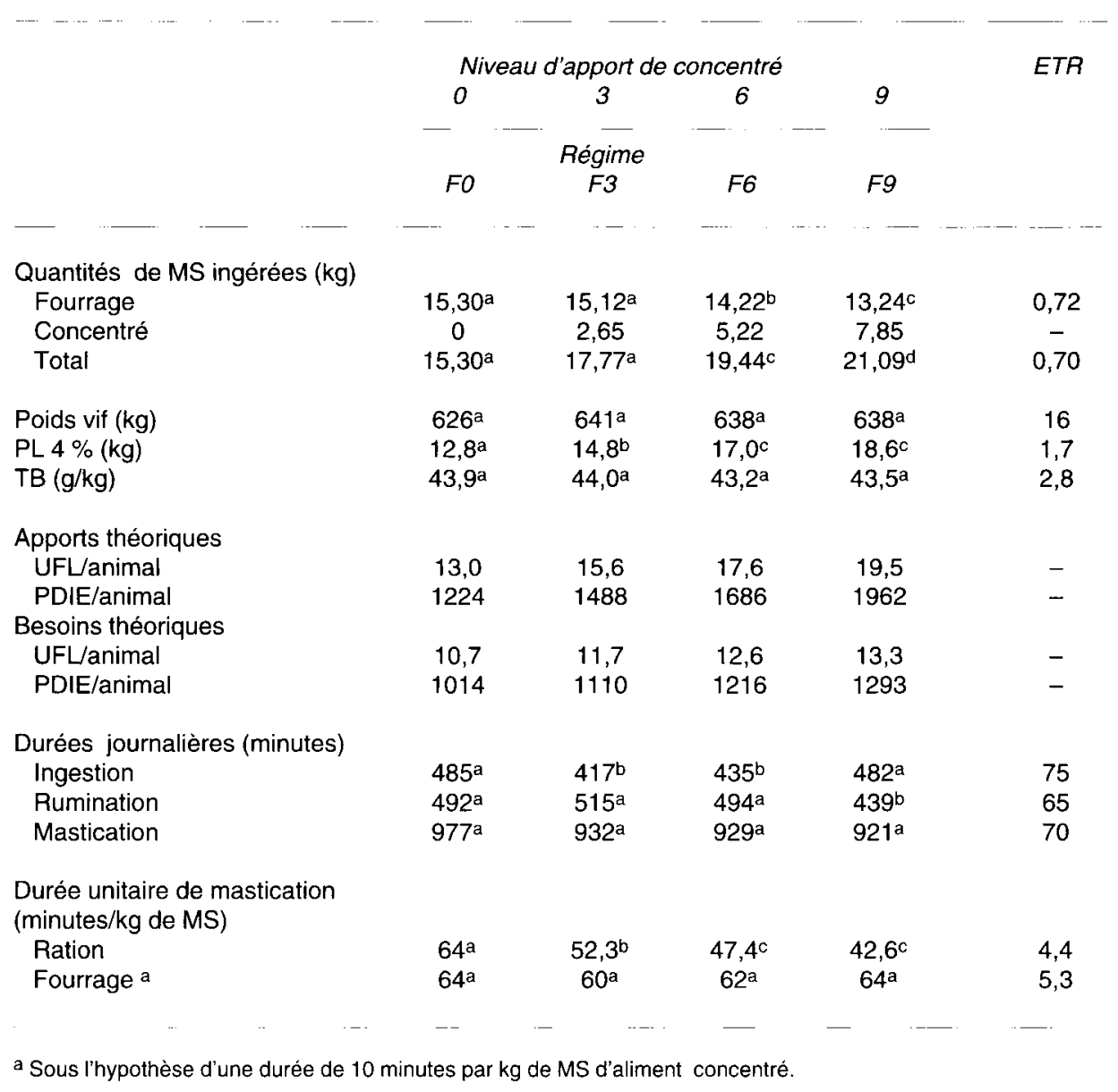

La relation [4] n'est pas améliorée si on considère QIT :

$$
\begin{aligned}
& \mathrm{IM}_{r}=72,6-0,64 \text { QIT }-44,6 \mathrm{C} \\
& ( \pm 13,1) \quad( \pm 0,84) \quad( \pm 14,5)[5] \\
& S \text { NS } S \\
& R^{2}=0,639 \text { ETR }= \pm 5,98
\end{aligned}
$$

\section{DISCUSSION ET CONCLUSION}

Par rapport aux résultats bien connus sur les taux de substitution (Dulphy et al, 1987 ;
Faverdin et al, 1991), ceux trouvés dans cet essai sont plutôt faibles. Ils indiquent une valeur alimentaire moyenne du fourrage utilisé malgré une bonne valeur nutritive prévue par les analyses. L'effet important de l'apport d'aliment concentré sur la production de lait confirme cette valeur moyenne. D'ailleurs pour le traitement FO la valorisation du kilo d'ensilage n'est que de $0,7 \mathrm{UF} / \mathrm{kg}$ de MS (besoins rapportés aux quantités ingérées).

Dans cet essai, l'indice de mastication de la ration diminue très nettement avec la 
proportion de concentré, avec une variation de 54 minutes lorsque la proportion de concentré passe de 0 à 1 , contre 28 minutes dans l'article de Sauvant et al (1990), compilation inter-essais. II ne varie pas avec la quantité ingérée totale contrairement aux résultats de Sauvant et al (1990) (pente = $-2,41$, contre $-0,64$ dans cet essai) et Faverdin (pente $=-1,34$; résultat non publié). Dans ces conditions on peut donc admettre que l'indice de mastication du fourrage est relativement indépendant du traitement étudié.

Par rapport aux valeurs publiées pour les ensilages d'herbe (Sudweeks et al, 1981 ; Dulphy et al, 1993), l'indice de mastication pour le fourrage étudié, de l'ordre de 63 minutes/ kg de MS, est relativement faible, plutôt comparable à celui des ensilages de maïs.

L'augmentation de l'apport de concentré aurait pu entraîner une augmentation de la durée unitaire de mastication du fourrage, par suite d'une baisse progressive de la vitesse de digestion (cf discussion de Dulphy et al, 1996), que l'animal compenserait par une mastication un peu plus poussée (Guérin et Dulphy, 1984). Cependant, dans cet essai, l'augmentation de la durée unitaire de mastication du fourrage avec la proportion de concentré apparaît comme nulle, ce qui rejoint les conclusions de Sudweeks et al (1975), Dulphy et al (1993) et de Jarrige et al (1995). II serait cependant intéressant de confirmer ces observations car la durée journalière de mastication du régime témoin Fo est un peu plus élevée que celle des trois autres. Jusque vers $40 \%$ d'aliment concentré dans la ration, il est donc tout à fait possible que les interactions concentré/fourrage sur l'indice de mastication des fourrages soient faibles, du moins pour des rations équilibrées en matières azotées, et contenant une proportion modérée d'amidon, comme dans cet essai.

II est très probable cependant qu'au-delà de $50 \%$ de concentré les interactions deviennent importantes. Ainsi dans un essai de Woodford et al (1986), en prenant une durée unitaire de mastication du concentré de 10 minutes, l'indice de mastication du foin de luzerne passe de 55 à 64 minutes $/ \mathrm{kg}$ de MS lorsque la proportion de concentré passe de 47 à $72 \%$. En outre, dans cet essai les vaches recevaient et avaient un niveau d'alimentation plus élevée que dans le nôtre.

En conclusion, il semble bien, sans que cela entraîne une erreur importante, qu'on puisse considérer comme additifs les indices de mastication des aliments distribués, tant que la proportion de concentré dans la ration n'est pas élevée. Quand cette dernière dépasse 40 à $50 \%$ l'indice de mastication du fourrage pourrait augmenter. Ces deux conclusions devront être cependant confirmées. Pour l'instant, le fait de considérer encore comme additifs les indices de mastication ne devrait pas avoir, pour autant, de conséquence fâcheuse car la durée journalière réelle de mastication des animaux a alors une forte probabilité d'être supérieure à celle prévue.

\section{RÉFÉRENCES}

Balch CC (1971) Proposal to use time spent chewing as an index of the extent to which diets for ruminants possess the physical property of fibrousness characteristic of roughages. Br J Nutr 26, 383-392

Dulphy JP, Demarquilly C, Henry M (1975) Perte de composés volatils lors de la détermination à l'étuve de la teneur en matière sèche des ensilages. Ann Zootech 24, 743-756

Dulphy JP, Faverdin P, Micol D, Bocquier F (1987) Révision du système des unités d'encombrement. Bull Tech CRZV Theix INRA, 70, 35-48

Dulphy JP, Jailler M, L'Hotelier L (1996) Fill effect of concentrate in the rumen of sheep. Ann Zootech (sous presse)

Dulphy JP, Rouel J, Jailler M, Sauvant D (1993) Données complémentaires sur les durées de mastication chez des vaches laitières recevant des rations riches en fourrage : influence de la nature du fourrage et du niveau d'apport d'aliment concentré. INRA Prod Anim 6, 297-302 
Faverdin P, Dulphy JP, Coulon JB, Vérité R, Garel JP, Rouel J, Marquis B (1991) Substitution of roughage by concentrates for dairy cows. Liv Prod Sci $27,137-$ 156

Guérin H, Dulphy JP (1984) Influence de l'apport complémentaire de maïs, de pulpe de betterave ou de mélasse sur la valeur alimentaire d'un foin. Ann. Zootech 33, 509-532

Jarrige R, Dulphy JP, Faverdin P, Baumont R, Demarquilly $C$ (1995) Activités d'ingestion et de rumination. Chapitre 4. In: Nutrition des ruminants domestiques ( $\mathrm{R}$ Jarrige, $\mathrm{Y}$ Ruckebush, $\mathrm{C}$ Demarquilly, $\mathrm{MH}$ Farce, M Journet, eds), 117-175

Journet M (1988) Optimisation des rations. In: Alimentation des bovins, ovins et caprins ( $R$ Jarrige, ed) Inra, 121-133

Leveillé M, Jouany JP, Brun JP (1979) Analyse automatique du comportement alimentaire et mérycique chez le mouton. Ann Biol Anim Bioch Biophys 19, (3B), 889-893

Nørgaard P (1990) The use of time spent chewing in the formutation of optimal rations for dairy cows. Paper presented at a meeting, Faculty of Veterinary
Medecine, Karl Maxx University, Leipzig, Allemagne, 6 December, $4 \mathrm{p}$

SAS Institute (1985) SAS User's Guide. SAS Institute Inc, Cary, NC, États-Unis

Sauvant D, Dulphy JP, Michalet-Doreau B (1990) Le concept d'indice de fibrosité des aliments des ruminants. INRA Prod Anim 3, 309-318.

Sudweeks EM, Ely LO, Mertens DR, Sisk LR (1981) Assessing minimum amounts and form of roughages in ruminant diets: roughage value index system. JAnim Sci 53, 1406-1411

Sudweeks EM, Mc Cullough ME, Sisk LR, Law SE (1975) Effects of concentrate type and level and forage type on chewing time of steers. J Anim Sci 41 , 219-224

Taniguchi K, Sugihara M, Yamatani Y, Otoni I (1986) Effects of starch supplementation on the fiber digestiblity and the chewing time of hay consumed by sheep. Jap J Zootech 57, 1046-1049

Woodford JA, Jorgensen NA, Barrington GP (1986) Impact of dietary fiber and physical form on performance of lactating dairy cows. J Dairy Sci 69,1035 1047 